\title{
Development of Numerical Tools for the Investigation of Plasma Detachment from Magnetic Nozzles
}

\author{
Kamesh Sankaran* \\ Whitworth College, Spokane, WA, 99251
}

\author{
Kurt A. Polzin ${ }^{\dagger}$ \\ NASA-Marshall Space Flight Center, Huntsville, AL 35812
}

\begin{abstract}
A multidimensional numerical simulation framework aimed at investigating the process of plasma detachment from a magnetic nozzle is introduced. An existing numerical code based on a magnetohydrodynamic formulation of the plasma flow equations that accounts for various dispersive and dissipative processes in plasmas was significantly enhanced to allow for the modeling of axisymmetric domains containing three-dimensional momentum and magnetic flux vectors. A separate magnetostatic solver was used to simulate the applied magnetic field topologies found in various nozzle experiments. Numerical results from a magnetic diffusion test problem in which all three components of the magnetic field were present exhibit excellent quantitative agreement with the analytical solution, and the lack of numerical instabilities due to fluctuations in the value of $\nabla \cdot B$ indicate that the conservative MHD framework with dissipative effects is well-suited for multi-dimensional analysis of magnetic nozzles. Further studies will focus on modeling literature experiments both for the purpose of code validation and to extract physical insight regarding the mechanisms driving detachment.
\end{abstract}

\section{Nomenclature}

$\mathbf{B}$
$\overline{\bar{B}}_{M}$
$E$
$E^{\prime}$
$\mathcal{E}$
$k$
$\mathbf{j}$

$\begin{array}{ll}p & \text { pressure, } \mathrm{Pa} \\ \mathbf{q} & \text { dissipative power per area, } \mathrm{W} / \mathrm{m}^{2} \\ T & \text { temperature, } \mathrm{K} \\ \mathbf{u} & \text { fluid velocity, } \mathrm{m} / \mathrm{s} \\ \eta & \text { resistivity, } \Omega \cdot \mathrm{m} \\ \mu_{\circ} & \text { permeability of free space, } \mathrm{H} / \mathrm{m} \\ \rho & \text { mass density, } \mathrm{kg} / \mathrm{m}^{3}\end{array}$

\section{Introduction}

M AGNETIC nozzles are used in many experiments to control - confine, cool, accelerate, and direct - plasma flows using magnetic fields in lieu of material boundaries. Studies of plasma detachment have been motivated by its role in many astrophysical phenomena ${ }^{1-3}$ and by the various terrestrial applications that use magnetic nozzles to exhaust power and particles. These applications include plasma flows in fusion systems ${ }^{4}$ where understanding detachment is important to controlling the thermal loads on components, rapid plasma cooling for laser emission where understanding recombination during the expansion is crucial to the generation of a population inversion, and directional control of a plasma jet in etching processes. ${ }^{5}$ We focus on the role an applied magnetic field and magnetic nozzle can play in facilitating the transfer of random thermal energy into directed kinetic energy in a plasma thruster.

Since magnetic fields are solenoidal, the design of a magnetic nozzle for a plasma thruster involves conflicting goals of confinement (leading to energy conversion and plasma acceleration along the magnetic field) and detachment (allowing for plasma motion transverse to the magnetic field leading to momentum transfer and thrust production). For a thruster it is important to understand the process of plasma detachment from an applied magnetic field both in terms of developing a capability to accurately model the process and for the development of a basic physical understanding of the process, with the end goal being a better understanding of thrusters that employ magnetic nozzles.

\footnotetext{
*Assistant Professor, Physics Department. Member AIAA.

†Propulsion Research Engineer, Nuclear Systems Branch, Propulsion Systems Department. Member AIAA.

This material is declared a work of the U.S. Government and is not subject to copyright protection in the United States.
} 
While there have been many experimental ${ }^{5-16}$ and analytical ${ }^{17-21}$ efforts to investigate detachment mechanisms, multidimensional numerical investigations have been sparse. ${ }^{22}$ Plasma properties in real devices can vary significantly in space and time. Such variations, as well as the complicated magnetic field topologies found in many nozzles, make analytical treatments of detachment difficult if not wholly intractable. In addition many atomic and plasma kinetic phenomena, such as the values of various transport coefficients or the non-ideal relationship between the pressure, density, and temperature as well as their reiationship with the ratio of specific heats in the plasma, cannot be easily measured with common experimental diagnostics but can greatly affect the detachment process. Consequently, numerical simulations that incorporate realistic models of the various plasma processes can play a unique role in developing an understanding of the mechanisms that cause detachment and learning how these processes scale with various plasma and applied field properties.

In this paper, we discuss the recent development of multidimensional numerical tools that can be used to investigate plasma detachment mechanisms. The numerical code is based on a conservation formulation of the magnetohydrodynamic (MHD) equations, and contains models for various classical and anomalous transport phenomena and a real (non-ideal) equation of state. The code was introduced in Ref. [23] and has been previously used to simulate self-field magnetoplasmadynamic thrusters (MPDT) ${ }^{24,25}$ and a lithium Lorentz force accelerator. ${ }^{26}$ The capabilities of the code are significantly enhanced over those previous studies, resulting in the capability to model plasma flows in magnetic nozzles. In addition, the code could also be used to simulate applied-field MPDTs, which incorporate the processes found in an MPDT with the magnetic nozzle field topology. We present results from a magnetic diffusion test case involving multi-dimensional magnetic fields. These results suggest that certain difficulties encountered when applying other MHD simulation codes to the magnetic nozzle problem may not arise in our numerical studies. In future studies the code will be employed to model various plasma flows that show potential propulsive benefits. The modeling results will be compared with available literature data to explain various experimental observations and gain insight into the various physical processes associated with plasma flow in a magnetic nozzle and plasma detachment.

The outline for the rest of this paper is as follows. In Sect. II we review various proposed mechanisms that could lead to plasma detachment. The physical model, governing equations, and numerical formulation for the nozzle simulations are described in Sect. III. We present the results of a plasma simulation test case involving multi-dimensional magnetic fields in Sect. IV, comparing the analytical solution to a magnetic diffusion problem with corresponding numerical simulation results.

\section{Review of Plasma Detachment Theories}

The problem of plasma detachment from a magnetic field has been addressed in various branches of plasma physics. A survey of the literature suggests several mechanisms that could lead to detachment. These are listed and discussed in turn below.

\section{A. Resistive Detachment}

A plasma that is sufficiently resistive can support collisionally driven plasma transport transverse to the magnetic field. ${ }^{20}$ Collisions that accomplish this arise either from classical Coulomb interactions between electrons and ions or through anomalous transport driven by interactions between electrons and electromagnetic waves (i.e. plasma turbulence ${ }^{27}$ ). Hooper ${ }^{20}$ analyzed detachment for such a case and concluded that the resistively-driven change in magnetic flux due to cross-field transport resulted in a violation of the "frozen-in" flow condition leading to a loss of magnetic flux over time given by,

$$
\frac{\psi(t)}{\psi(0)} \simeq \exp \left(-\int \frac{\epsilon_{\circ} \eta \omega_{p}^{2}}{u} d l\right) \simeq \exp \left(-\int \frac{\nu_{\text {coll }}}{u} d l\right),
$$

where $\psi$ is the magnetic flux, $\epsilon_{\circ}$ is the permittivity of free space, $\eta$ is the plasma resistivity, $\omega_{p}$ is the plasma frequency, $u$ is the plasma velocity, and $\nu_{\text {coll }}$ is the effective electron collision frequency. This.relationship can be used to estimate the minimum required collision frequency for plasma detachment.

\section{B. Kinetic Detachment}

Studies of solar wind in the late 1950s led to the proposition that plasma detaches from a magnetic field when the flow velocity exceeds the local Alfven speed. ${ }^{28}$ This corresponds to the case where the kinetic energy of the plasma is greater than the magnetic field energy. ${ }^{29}$ As the plasma expands in the downstream portion of a magnetic nozzle; the 
ratio of plasma kinetic energy to the energy in the magnetic field continues to increase. This ratio can also be written as the square of the ratio of plasma velocity to the Alfvèn speed $v_{A}$,

$$
\beta=\frac{\rho u^{2} / 2}{B^{2} /\left(2 \mu_{\circ}\right)}=\left(\frac{u}{B / \sqrt{\mu_{\circ} \rho}}\right)^{2}=\left(\frac{u}{v_{A}}\right)^{2}=M_{A}^{2} .
$$

When $\beta$ exceeds 1 , it is theorized that plasma will possess sufficient kinetic energy to permit detachment from the magnetic field. This theory was recently applied to plasma thrusters by Arefiev and Breizman. ${ }^{21}$ Using MHD theory, they concluded that plasma would detach from the nozzle, even in the absence of collisions, with an detachment efficiency (ratio of the outgoing to incoming axial momentum fluxes) $\eta_{d}$ given by,

$$
\eta_{d} \sim 1-\left(\frac{\theta_{\circ}}{2}\right)^{2}-\left(\frac{v_{A}}{\sqrt{2} u}\right)^{2}
$$

where $\theta_{\circ}$ is the nozzle divergence angle.

\section{Recombination Detachment}

Applied magnetic field confinement of particles in an expanding jet is lost when the recombination rate is such that ionized particles are quickly converted into neutrals. ${ }^{30}$ However, based on expansion and cooling rates in typical thruster flows, it appears unlikely that the recombination rate would be high enough to support this detachment mechanism. It has been suggested ${ }^{30}$ that injecting a cold gas of heavy molecules (such as $\mathrm{Kr}$ ) into the expanding plasma flow for the purposes of cooling the electrons and enhancing detachment could lead to enhanced recombination-driven detachment.

\section{Non-Adiabatic Detachment}

If a magnetic field has a strong spatial gradient, the condition of adiabatic invariance of a charged particle's magnetic moment may not hold. Specifically, if the ratio of a particle's gyro radius to the characteristic length scale of change in the magnetic field, $r_{g} /(B / \nabla B)$, is greater than unity then charged particles are not confined to follow magnetic field lines through invariance of their magnetic moments and detachment can occur. In the types of plasmas found in magnetic nozzles, this ratio could be greater than unity for ions but is typically much less than unity for electrons. With electrons confined to magnetic field lines, it is unlikely that this detachment mechanism plays a major role in propulsive magnetic nozzles since the plasma expelled in these systems is typically quasineutral. ${ }^{20}$

\section{E. Electron Inertia Detachment}

It was proposed that because electrons possess finite mass, they can inhibit the flow of azimuthal currents in the nozzle leading to collisionless detachment. ${ }^{17,19}$ A theoretical treatment showed that plasma detachment could occur when the non-dimensional parameter,

$$
G \simeq \omega_{e} \omega_{i}\left(\frac{r_{\mathrm{o}}}{u_{\mathrm{o}}}\right)^{2}
$$

was below a critical value. Here $\omega_{e}$ and $\omega_{i}$ are the gyro frequencies of the electrons and ions, and $r_{\circ}$ and $u_{\circ}$ are the radius and velocity of the plasma jet at the throat. This effect, however, is quite minimal and could be taken to represent a lower bound on the amount of detachment possible in a given experiment.

\section{Physical Models for Magnetic Nozzle Simulations}

Among the abovementioned detachment theories, the resistive and kinetic mechanisms are most amenable to investigation using models based on the continuum treatment of plasma flows. An analysis of detachment driven by recombination, non-adiabaticity, or electron inertia requires either a particle-in-cell (PIC) or hybrid treatment of the plasma. The numerical framework we describe below is based on the MHD (continuum) formulation of the plasma flow governing equations, restricting our studies of detachment to the resistive and kinetic detachment mechanisms. 


\section{A. MHD Equations}

The governing equations for an MHD flow problem represent the conservation relations for mass, momentum, magnetic flux, and energy. These can be compactly written in the form,

$$
\frac{\partial}{\partial t}\left[\begin{array}{c}
\rho \\
\rho \mathbf{u} \\
\mathbf{B} \\
\mathcal{E}
\end{array}\right]+\nabla \cdot\left[\begin{array}{c}
\rho \mathbf{u} \\
\rho \mathbf{u u}+\overline{\bar{p}}-\overline{\overline{\mathcal{B}}}_{M} \\
\mathbf{u B}-\mathbf{B} \mathbf{u} \\
(\mathcal{E}+p) \mathbf{u}-\overline{\overline{\mathcal{B}}}_{M} \cdot \mathbf{u}
\end{array}\right]=\mathbf{D}
$$

The right hand side of the equation, $D$, contains dissipative effects, which are also written as the divergence of fluxes. ${ }^{26}$

The compact vector-tensor equations are expanded into their individual vector components and rewritten in a finite-volume form so that they can be solved by a conservative MHD code. The expanded equation set, assuming no azimuthal variation $(\partial / \partial \theta=0)$, is:

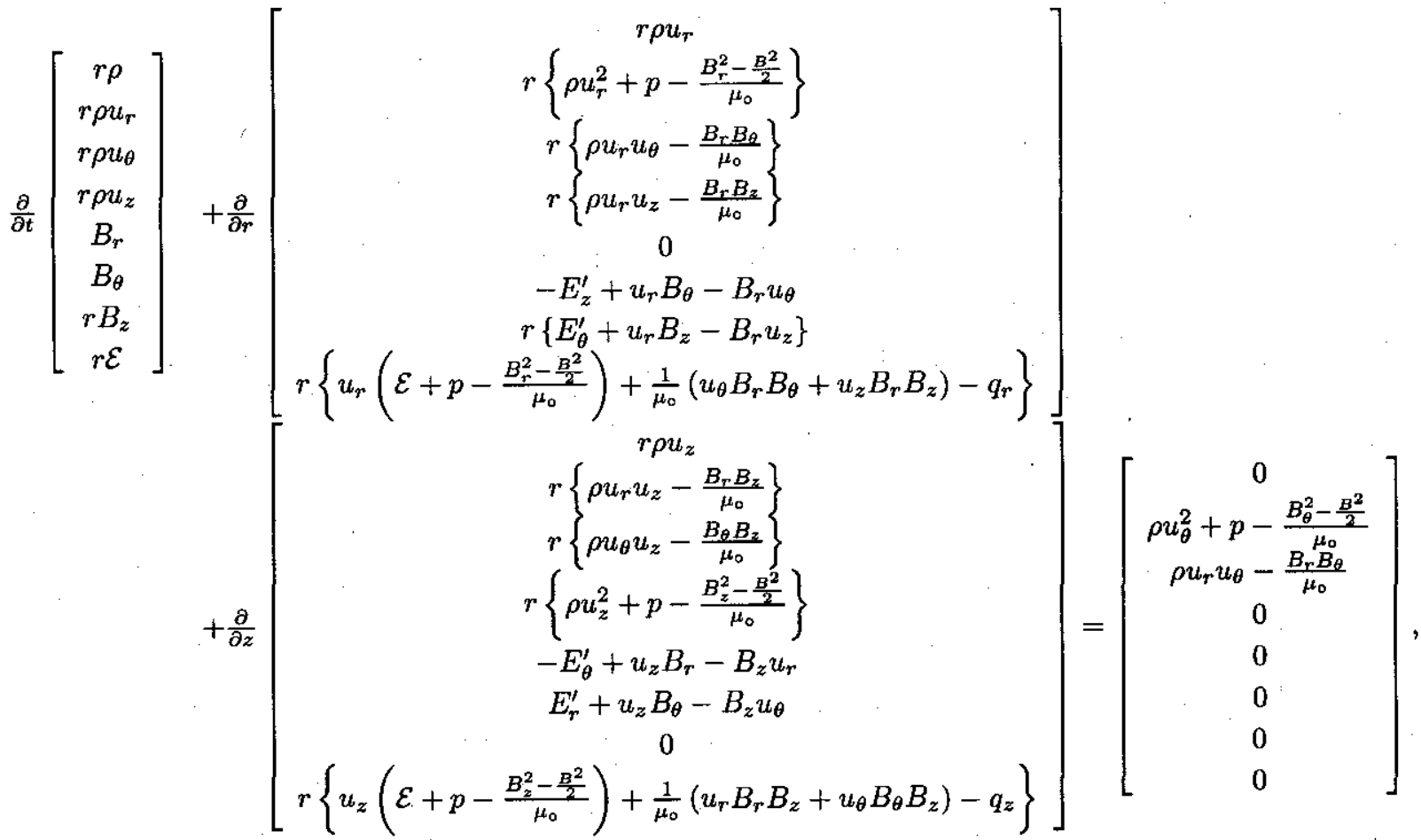

where

$$
\begin{aligned}
& q_{r}=\left(E_{z}^{\prime} B_{\theta}-E_{\theta}^{\prime} B_{z}\right) / \mu_{\circ}+\left(k_{r r} \partial T_{e} / \partial r\right)+\left(k_{r z} \partial T_{e} / \partial z\right) \\
& q_{z}=\left(E_{r}^{\prime} B_{\theta}-E_{r}^{\prime} B_{\theta}\right) / \mu_{\circ}+\left(k_{z r} \partial T_{e} / \partial r\right)+\left(k_{z z} \partial T_{e} / \partial z\right)
\end{aligned}
$$

In the case of an applied magnetic field, the components $B_{r}$ and $B_{z}$ are the sum of the steady-state applied field and an induced field that is initially time-varying but eventually converges to a steady-state value. The set of MHD equations are closed by the generalized Ohm's law,

$$
\mathbf{E}^{\prime}=\mathbf{E}+(\mathbf{u} \times \mathbf{B})=\eta \mathbf{j}+\frac{(\mathbf{j} \times \mathbf{B})-\nabla p_{e}}{e n_{e}},
$$

and Ampère's law. The models for transport coefficients, equation-of-state, ionization and thermal nonequilibrium are described in Ref. [26]. Implementation of the entire equation set given in Eqns. (6) represents a significant enhancement in simulation capabilities relative to the previous work by Sankaran et al. ${ }^{24-26}$ where $B_{r}, B_{z}$, and $u_{\theta}$, and non-conserved, secondary (calculated) quantities $j_{\theta}$ and $E_{\theta}$, were all zero. 


\section{B. Finite Volume Formulation}

The solution of Eqs. (6) in a conservative manner is performed by writing each equation in their integral form. All eight conservation laws (mass, three components of momentum, three components of magnetic flux, and total energy) can be individually integrated over the cell area,

$$
\iint_{A}\left[\frac{\partial U}{\partial t}=\left(\frac{\partial F_{r}}{\partial r}+\frac{\partial F_{z}}{\partial z}\right)+\text { Source }\right] d A
$$

A vector $\hat{\mathbf{F}}=\left[F_{z},-F_{r}\right]$ is defined such that

$$
\nabla \times\left.\hat{\mathbf{F}}\right|_{\theta}=\frac{\partial \hat{F}_{r}}{\partial z}-\frac{\partial \hat{F}_{z}}{\partial r}=\frac{\partial F_{r}}{\partial r}+\frac{\partial F_{z}}{\partial z} .
$$

This definition allows us to write the right hand side of Eq. (8) as the curl of $\hat{\mathbf{F}}$. Using Stokes' theorem, the curl over the cell area can be expressed as the line integral around the edges of the cell,

$$
\iint_{A} \frac{\partial U}{\partial t} d A=\oint \hat{\mathbf{F}} \cdot \mathbf{d l}+\iint_{A}(\text { Source }) d A .
$$

Since this equation is true irrespective of the shape of the control volume, the fluxes $F_{r}$ and $F_{z}$ can written in the $(r, z)$ coordinate system, as shown in Eqs. (6), regardless of the shape of the cell.

\section{Magnetostatic Model}

A separate magnetostatic solver ${ }^{31}$ is used to model the applied magnetic field topology of the experiment being simulated. The magnetic coils in these experiments have tens of thousands of loops, while the coils in magnetostatic simulation had only a handful of loops. Consequently, the primary goal of our magnetostatic modeling effort was to recreate a "reasonable/realistic" topology.

We have modeled the applied magnetic field from the magnetic nozzle experiments described in Refs. [5-15, 22, 32]. In Fig. 1 we show the nozzle topology for the experiment described by Kuriki and Okada ${ }^{6}$ generated using the magnetostatic solver. The computationallygenerated field was compared to published values from

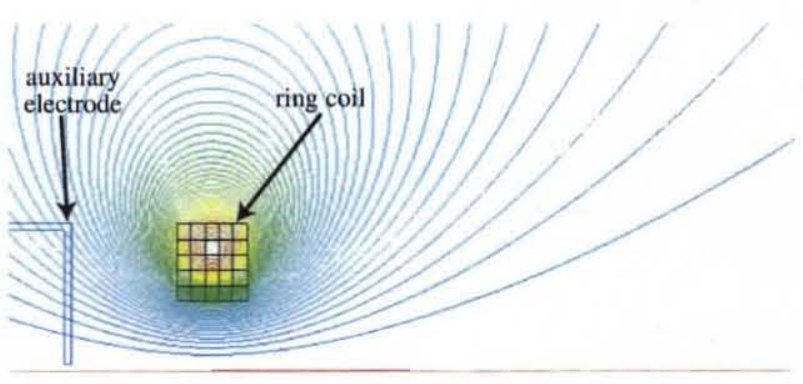

Figure 1. Magnetostatic simulation of flux lines in the $r-z$ plane of the nozzle in Ref. [6]. the experimental setup. Despite our simplifying the problem by using only a few magnet coil loops, the magnetostatic solver produced a magnetic field topology possessing field values that were within a few percent of those measured. ${ }^{6}$

The magnetostatic solver ${ }^{31}$ outputs the relevant variables $\left(B_{r}, B_{z}\right)$ on an orthogonal $(r, z)$ grid. An interpolation script was used to transfer the magnetic field data onto the non-orthogonal grid used by the MHD code.

\section{Code Validation}

Under the assumptions of very low magnetic Reynolds number and isotropic, constant plasma resistivity, Faraday's law reduces to a magnetic diffusion problem governed by the equation

$$
\frac{\partial \mathbf{B}}{\partial t}=\frac{\eta}{\mu_{\circ}} \nabla^{2} \mathbf{B} .
$$

We consider the case of a plasma in the presence of a constant solenoidal field in the $r-z$ plane. At time $t=0$ a nonuniform current $\mathbf{j}=j_{z}(r) \hat{\mathbf{z}}$ is introduced into the plasma. The current resistively diffuses until it is uniform, and the induced magnetic field (in the $\hat{\theta}$ direction) due to the current relaxes to its equilibrium value. Assuming axisymmetry and no variation of $B_{\theta}$ in the $z$-direction, the resistive diffusion of the azimuthal, induced magnetic field is given by

$$
\frac{\partial B_{\theta}}{\partial t}=\frac{\eta}{\mu_{\circ}}\left(\frac{\partial^{2} B_{\theta}}{\partial r^{2}}+\frac{1}{r} \frac{\partial B_{\theta}}{\partial r}-\frac{B_{\theta}}{r^{2}}\right) .
$$


We suppose that the initially non-uniform current produces a magnetic field

$$
B_{\theta}(r, 0)=\frac{3 \mu_{\circ} I}{4 \pi R^{2}} r\left(1-\frac{r^{2}}{3 R^{2}}\right)
$$

where $R$ is the outer radius of the domain and $I$ is the total current. The boundary conditions on $B_{\theta}$ at $r=0$ and $r=R$ are

$$
\begin{aligned}
B_{\theta}(0, t) & =0 \\
B_{\theta}(R, t) & =\frac{\mu_{\mathrm{o}} I}{2 \pi R} .
\end{aligned}
$$

The time-dependent solution for the induced magnetic field is

$$
B_{\theta}(r, t)=\left[\frac{\mu_{\circ} I}{2 \pi R^{2}} r\right]+\left[\sum_{n=1}^{\infty}\left\{P_{n} J_{1}\left(\frac{z_{n}}{R} r\right)\right\} \exp \left(-\left(\frac{z_{n}}{R}\right)^{2} \frac{\eta}{\mu_{\circ}} t\right)\right],
$$

where

$$
P_{n}=\left(\int_{0}^{R} g(r) \cdot r \cdot J_{1}\left(\frac{z_{n}}{R} r\right) d r\right) /\left(\int_{0}^{R} J_{1}\left(\frac{z_{n}}{R} r\right) \cdot r \cdot J_{1}\left(\frac{z_{n}}{R} r\right) d r\right)
$$

and

$$
g(r)=B_{\theta}(r, 0)-\frac{\mu_{\circ} I}{2 \pi R^{2}} r
$$

Here $J_{1}$ is the Bessel function of the first kind and $z_{n}$ are its zeros. We found that the summation given in Eq. (16) could be truncated at $n=5$ without a loss in accuracy.

In our test case, $I=10^{4} \mathrm{~A}, R=0.05 \mathrm{~m}$ and $\eta=$ $10^{-4}$ R.m. The plasma density was chosen to be high ( $n_{e}=n_{i}=5 \times 10^{22} \mathrm{~m}^{-3}$ ) so that diffusion processes would dominate convection and would thus enable us to compare the results of the simulation with diffusion theory. We simulated the test case by itself, against a uniform axial magnetic field background, and against the solenoidal applied magnetic field (with components $B_{r}$ and $B_{z}$ ) given in Fig. 1. The solution in Eq. (16) is unaffected by an applied, purely axial magnetic field, and the numerical solutions exhibit good agreement in this case. For the solenoidal field, the radial component is exactly zero at the axial plane that bisects the ring coil. A comparison between the numerical results for $B_{\theta}$ on the axial plane where $B_{r}=0$ and the analytical solutions at different instances in time are presented in Fig. 2.

From the figure, we observe that the code accurately models the time-dependent evolution of the azimuthal magnetic field and the magnetic field components $B_{r}$ and $B_{z}$ (from Fig. 1) remain unperturbed. While the temporal evolution of $B_{\theta}$ along the axial plane bisecting the ring coil was generated against the solenoidal applied field, it is worth noting that the numerical and analytical solutions were in agreement for the other two applied magnetic field test cases (no field and a purely axial applied field).

Another important result is that the code handled a three-dimensional $\mathbf{B}$-field without noticeable deviation in the value of $\nabla \cdot \mathbf{B}$ from zero. Without any assumptions on $\nabla \cdot \mathrm{B}$, Faraday's law is,
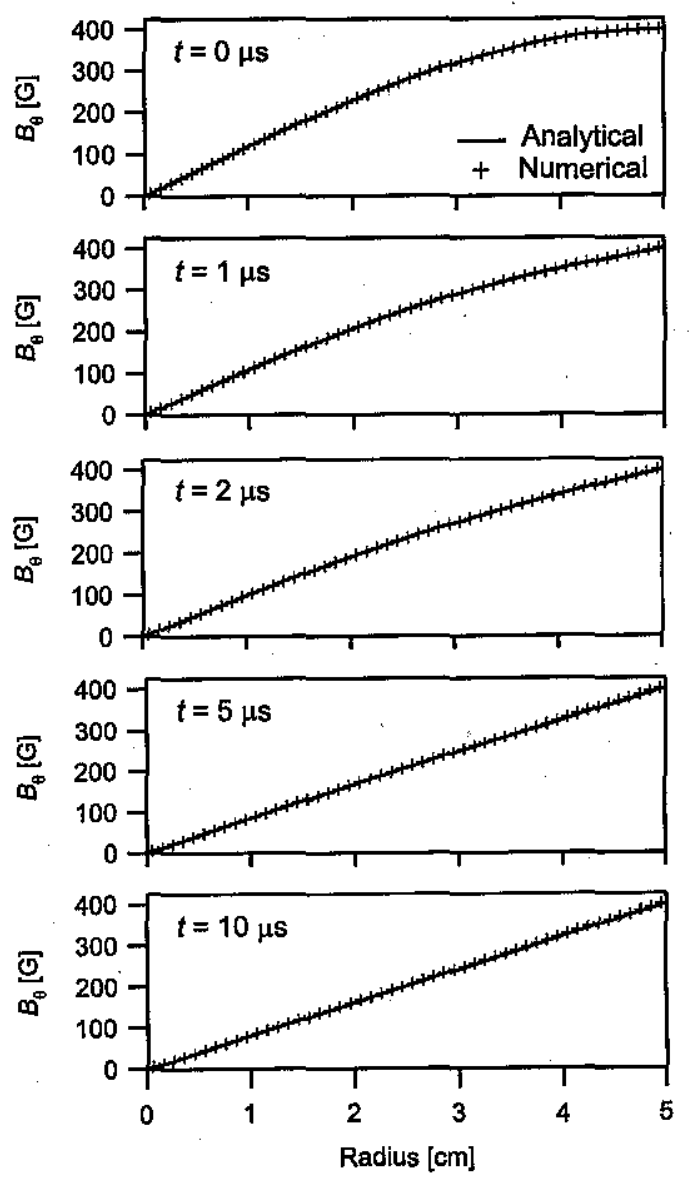

Figure 2. Comparison between analytical and numerical solutions for $B_{\theta}$ in the magnetic diffusion test case. Numerical solutions were calculated against the magnetic field given in Fig. 1. 


$$
\frac{\partial \mathbf{B}}{\partial t}=-\nabla \times \mathbf{E}-(\nabla \cdot \mathbf{B}) \mathbf{u} .
$$

While it is physically true that $\nabla \cdot \mathbf{B} \equiv 0$, it is often not true numerically owing to truncation and round-off errors. In fact, this has been a source of difficulty in extending MHD codes to three-dimensional vector fields since these small deviations in the divergence can lead to numerical instabilities that are unbounded. ${ }^{33}$ Consequently, a significant amount of work has gone into developing MHD solution algorithms that strictly enforce the condition $\nabla \cdot \mathbf{B} \equiv 0$. In our case, because the second term on the right-hand side of Eq. (19) is small, owing to the fact that both $\nabla \cdot \mathbf{B}$ and $|\mathbf{u}|$ are small, truncation errors do not significantly affect the solution to the diffusion problem.

Many previous MHD codes ${ }^{34,35}$ have employed the ideal-MHD assumption because they were developed for the purpose of simulating problems with high magnetic Reynolds number - either due to very low resistivity (as is the case in fusion studies) or due to the dominance of convection (as is the case in many space and astrophysical plasmas). In such cases the absence of any significant dissipation allows for the growth of numerical oscillations ${ }^{33}$ due to errors in the calculation of $\nabla \cdot \mathbf{B}$. Fortunately, in many plasma thrusters and magnetic nozzles employed for propulsive purposes, the magnetic Reynolds number $R e_{m} \sim \mathcal{O}(1)$ and the plasma's resistivity provides a dissipative mechanism within the domain that can damp the numerical instabilities in much the same way that viscosity damps instabilities in the solution of the momentum equation.

\section{Conclusions and Future Work}

We have presented the formulation of a simulation tool that is capable of supporting multidimensional numerical studies of plasma flows and detachment in magnetic nozzles. The code is based on a conservation formulation of the MHD governing equations and contains realistic models of various physical and transport phenomena. The applied magnetic field solution is generated using a separate magnetostatic solver. This field is used as an input for the plasma flow modeling. There are several proposed mechanisms that could drive the detachment process; the continuum-based formulation of the numerical problem limits us to the study of the resistive and kinetic detachment mechanisms. The code has demonstrated the ability to model a plasma problem in which all three components of the magnetic field were present, and during this simulation deviations from the condition $\nabla \cdot \mathbf{B}=0$ were small.

In a future paper, we shall describe simulations of a plasma flow in a magnetic nozzle. For validation purposes, we shall base our simulations on experiments described in Refs. [5-15, 22, 32]. Aside from code validation, the primary purposes of these and other future simulations are to:

- Investigate the feasibility of contouring the magnetic fields such that the plasma accelerates to super-Alfvénic speeds and detaches,

- Vary the plasma properties to investigate whether resistive or kinetic detachment occurs,

- Generate magnetic field topologies that might more readily lead to plasma detachment,

- Prescribe guidelines and scaling parameters with relevance to spacecraft propulsion magnetic nozzle design.

\section{Acknowledgments}

The authors thank Preston Hoffman for his assistance with the magnetostatic modeling. Support for Prof. Sankaran provided by the M. J. Murdock Charitable Trust and Whitworth College. This work performed under NASA contract SAA8-05968 and supported by the MSFC Technology Transfer Office and the Exploration Systems Mission Directorate.

\section{References}

${ }^{1}$ R.M. Kulsrud, "Important PJasma Problems in Astrophysics," Physics of Plasmas, 2:1735, 1995.

${ }^{2}$ J.F. Drake, "Magnetic Explosions in Space," Nature, 410:525, 2001.

${ }^{3}$ X.H. Deng and H. Matsumoto, "Rapid magnetic reconnection in the Earth's magnetosphere mediated by whistler waves," Nature, 410:557, 2001.

${ }^{4}$ E.M. Hollman, A.Yu. Pigarov, R. Seraydarian, D.G. Whyte, and S.I. Krasheninnikov, "Particle balance measurements during detachment in a gas-target divertor simulator," Physics of Plasmas, 9(4):1226, 2002.

${ }^{5}$ K.F. Schoenberg, R.A. Gerwin, R.W. Moses, Jr., J.T. Scheuer, and H.P. Wagner, “Magnetohydrodynamic flow physics of magnetically nozzled plasma accelerators with applications to advanced manufacturing," Physics of Plasmas, 5(5):2090, 1998. 
${ }^{6} \mathrm{~K}$. Kuriki and O. Okada, "Experimental Study of a Plasma Flow in a Magnetic Nozzle," Physics of Fluids, 13(9):2262, 1970.

${ }^{7} \mathrm{~K}$. Kuriki and M. Inutake, "Super-Alfvénic flow and collision free shock wave in a plasma wind tunnel," Physics of Fluids, 17(1):95, 1974.

${ }^{8}$ T.M. York, The effects of magnetic nozzle configurations on plasma thrusters, Technical Report AAE Report AARL-P-90-1, Ohio State University/NASA Lewis Research Center, 1990.

${ }^{9}$ R.A. Gerwin, G.J. Marklin, A.G. Sgro, and A.H. Glasser, Characterization of plasma flow through magnetic nozzles, Technical Report LA-UR-89-4212, Los Alamos National Laboratory, 1989.

${ }^{10}$ K.F. Schoenberg, R.A. Gerwin, I. Henins, R.M. Mayo, J.T. Scheuer, and G.A. Wurden, "Preliminary investigation of power flow and performance phenomena in a multimegawatt coaxial plasma thruster," IEEE Transactions on Plasma Science, 21(6):625, 1993.

${ }^{11}$ D.C. Black, R.M. Mayo, R.A. Gerwin, K.F. Schoenberg, J.T. Scheuer, R.P. Hoyt, and I. Henins, "Two-dimensional magnetic field evolution measurements and plasma speed estimates from the coaxial thruster experiment," Physics of Plasmas, 1(9):3115, 1994.

${ }^{12}$ J.T. Scheuer, K.F. Schoenberg, R.A. Gerwin, R.P. Hoyt, I. Hennis, D.C. Black, R.M. Mayo, and R.W. Moses Jr., "A Magnetically-Nozzled, Quasi-Steady, Multimegawatt, Coaxial Plasma Thruster," IEEE Transactions on Plasma Science, 22(6):1015, 1994.

${ }^{13}$ R.P. Hoyt, J.T. Scheuer, K.F. Schoenberg, R.A. Gerwin, R.W. Moses Jr., I. Hennis, D.C. Black, and R.M. Mayo, "Optimization of Magnetic Nozzles for Coaxial Plasma Thrusters," 30th AIAA/ASME/SAE/ASEE Joint Propulsion Conference, Indianapolis, IN, June 1994. AIAA Paper 94-2992.

${ }^{14}$ R.P. Hoyt, "Magnetic Nozzle Design for Coaxial Plasma Accelerators," IEEE Transactions on Plasma Science, 23(3):481, 1995.

${ }^{15}$ D.C. Black, R.M. Mayo, and R.W. Caress, "Direct magnetic field measurement of flow dynamics in magnetized coaxial accelerator channels," Physics of Plasmas, 4(8):2820, 1997.

${ }^{16}$ D.G. Chavers et al, "Status of magnetic nozzle and plasma detachment experiment," AIP Conference Proceedings, 813:465, Jan. 2006.

${ }^{17}$ J.C. Sercel, "A Simple Model of Plasma Acceleration in a Magnetic Nozzle," 21st International Electric Propulsion Conference, Orlando, FL, July 1990. AIAA Paper 90-2597.

${ }^{18}$ R.W. Moses, R.A. Gerwin, and K.F. Schoenberg, "Resistive Plasma Detachment in Nozzle Based Coaxial Thrusters," AIP Conference Proceedings, 246:1293, Jan. 1992.

${ }^{19}$ D.A. Kaufman, D.G. Goodwin, and J.C. Sercel, "Plasma Separation from Magnetic Field Lines in a Magnetic Nozzle," 3 Ist AIAA Aerospace Sciences Meeting, Reno, NV, Jan. 1993. AIAA Paper 93-0817.

${ }^{20}$ E.B. Hooper, "Plasma Detachment from a Magnetic Nozzle," Journal of Propulsion and Power, 9(5):757, 1993.

${ }^{21}$ A.V. Arefiev and B.N. Breizman, "Magnetohydrodynamic scenario of plasma detachment in a magnetic nozzle," Physics of Plasmas, 12:043504, 2005.

${ }^{22}$ A.V. Ilin, F.R. Chang-Diaz, J.P. Squire, A.G. Tarditi, B.N. Breizman, and M.D. Carter, "Simulations of Plasma Detachment in VASIMR," 40th AIAA Aerospace Sciences Meeting, Reno, NV, Jan. 2002. AIAA Paper 2002-0346.

${ }^{23}$ K. Sankaran and E.Y. Choueiri, "An Accurate Characteristics-Splitting Scheme for Numerical Solution of MHD Equations," 26th International Electric Propulsion Conference, Kitakyushu, Japan, Oct. 1999. IEPC Paper 99-208.

${ }^{24}$ K. Sankaran, L. Martinelli, S.C. Jardin, and E.Y. Choueiri, "A Flux-Limited Numerical Method for the MHD Equations to Simulate Propulsive Plasma Flows," International Journal of Numerical Methods in Engineering, 53(5):1415, 2002.

${ }^{25}$ K. Sankaran, E.Y. Choueiri, and S.C. Jardin, "Comparison of Simulated Magnetoplasmadynamic Thruster Flowfields to Experimental Measurements," Journal of Propulsion and Power, 21(1):129, 2005.

${ }^{26} \mathrm{~K}$. Sankaran, Simulation of Plasma Flows in Self-Field Lorentz Force Accelerators, PhD thesis, Princeton Univ., Princeton, NJ, 2005.

${ }^{27}$ X.Q. Xu, W.M. Nevins, T.D. Rognlien, R.H. Bulmer, M. Greenwald, A. Mahdavi, L.D. Pearlstein, and P. Snyder, "Transitions of turbulence in plasma density limits," Physics of Plasmas, 10(5):1773, 2003.

${ }^{28}$ E.N. Parker, "Dynamics of the Interplanetary Gas and Magnetic Fields," Astrophysical Journal, 128:664, 1958.

${ }^{29}$ W.H. Ness, Introduction to Space Science, Gordon and Breach Science Publishers, 1965.

${ }^{30}$ G.I. Dimov and S.Yu. Taskaev, "Simulation of a supersonic plasma jet with recombination in a magnetic nozzle," 27th European Physical Society Conference on Controlled Fusion and Plasma Physics, ECA Vol. 24B:464, 2000.

${ }^{31}$ Ansoft Corporation, "Maxwell User Guide," http://www.ansoft.com/, 2002.

${ }^{32}$ F.R. Chang-Diaz, J.P. Squire, R.D. Bengston, B.N. Breizman, F.W. Baity, and M.D. Carter, "The Physics and Engineering of the VASIMR Engine," 36th AIAA/ASME/SAE/ASEE Joint Propulsion Conference, Huntsville, AL, July 2000. AIAA Paper 2000-3756.

${ }^{33}$ J.U. Brackbill and D.C. Barnes, "The effect of nonzero $\nabla \cdot \mathbf{B}$ on the numerical solution of the magnetohydrodynamic equations," Journal of Computational Physics, 35(3):426, 1980.

${ }^{34}$ K.G. Powell, P.L. Roe, T.J. Linde, T.I. Gombasi, and D.L. De Zeeuw, "A solution-adaptive upwind scheme for ideal magnetohydrodynamics," Journal of Computational Physics, 154(2):284, 1999.

${ }^{35}$ T.A. Gardiner and J.M. Stone, "An unsplit Godunov method for ideal MHD via constrained transport," Journal of Computational Physics, 205(2):509, 2005. 